\title{
Influence of Chemical Reaction, Heat Source, Soret and Dufour Effects on Separation of a Binary Fluid Mixture in MHD Natural Convection Flow in Porous Media
}

\author{
B.R.Sharma \\ Department of Mathematics \\ Dibrugarh University-786004 \\ Dibrugarh, Assam, India
}

\author{
Kabita Nath \\ Department of Mathematics \\ Dibrugarh University-786004 \\ Dibrugarh, Assam, India
}

\author{
Debozani Borgohain \\ Department of Mathematics \\ Dibrugarh University-786004 \\ Dibrugarh, Assam, India
}

\begin{abstract}
Separation of a heat absorbing or generating binary fluid mixture of incompressible viscous fluids in MHD natural convection flow about a vertical surface embedded in a saturated porous medium subjected to a chemical reaction is numerically analyzed, by taking into account the Soret and Dufour effects. The temperature and concentration profiles are drawn and the effects of magnetic, Lewish, Dufour, Soret, heat source, sustention parameter, order of the chemical reaction and chemical reaction parameter are exhibited graphically. The Nusselt and local Sherwood numbers are tabulated for various values of the above mentioned parameters. It is observed from the table that the Nusselt and local Sherwood numbers are effected significantly due to the presence of the chemical reaction and the magnetic field.
\end{abstract}

\section{Keywords}

Separation, binary fluid mixture, Soret effect, Dufour effect, chemical reaction, heat source, magnetic field and porous media

\section{INTRODUCTION}

Due to many important engineering and geophysical applications, coupled heat and mass transfer by natural convection in a fluid-saturated porous medium has attracted considerable attention in the last several decades. Nield and Bejan [1] and Ingham and Pop [2, 3] have made comprehensive reviews in this field. Several researchers such as Bejan and Hair [4], Makinde [5], Makinde and Ogulu [6], Ibrahim and Makinde [7] have studied free convection heat and mass transfer problems neglecting Dufour and Soret effects on the basis that they are of a smaller order of magnitudes.

Soret effect corresponds to species differentiation developing in an initial homogeneous mixture submitted to a thermal gradient [8]. The energy flux caused by a composition gradient is called Dufour or diffusion-thermo effect. Kafoussias and Williams [9], Anghel et al. [10], Postelnicu [11], Alam and Rahman [12] discussed free/forced convective heat and mass transfer boundary layer flow by taking into consideration the Dufour and Soret effects. Sharma [13] has analyzed the Soret and Dufour effects on demixing the binary fluid mixture in MHD natural convection flow in porous media.

The combined heat and mass transfer problems with chemical reaction are of great importance in several processes. The effect of a chemical reaction depends upon whether the reaction is homogeneous or heterogeneous. In well-mixed systems, the reaction is heterogeneous, if it takes place at an interface and homogeneous, if it takes place in solution. In most cases of chemical reactions, the reaction rate depends on the concentration of the species itself. A reaction is said to be of the order $\mathrm{n}$, if the reaction rate is proportional the $\mathrm{nth}$ power of concentration.

Chambre and Young [14] have analyzed a first order chemical reaction in the neighbourhood of a horizontal plate. Apelblat [15] studied analytical solution for mass with a chemical reaction of first order. Das et al. [16] have studied the effect of homogeneous first order chemical reaction on the flow past an impulsively started infinite vertical plate with uniform heat flux and mass transfer. Again mass transfer effects on moving isothermal vertical plate in the presence of chemical reaction by Das et al. [17]. Postelnicu [18] has studied the influence of chemical reaction on heat and mass transfer in porous media considering Soret and Dufour effects. A. Jyothi Bala and S. Vijaya Kumar Varma also studied effect of heat generation and homogeneous chemical reaction on unsteady MHD heat and mass transfer through a semi-infinite vertical plate [19].

The present paper deals with the two dimensional steady, laminar, heat absorbing or generating, MHD boundary layer flow of an incompressible viscous electrically conducting binary fluid mixture in a porous medium under the influence of magnetic field and in the presence of a chemical reaction. The effects of material parameters on temperature and concentration are discussed through graphs.

\section{MATHEMATICAL FORMULATION}

We consider the steady two - dimensional natural convection in a porous medium saturated with a Newtonian fluid mixture bounded by an impervious vertical stationary flat plate in presence of heat generation or absorption effects and magnetic field intensity applied in a direction perpendicular to the plate. The $\mathrm{x}$ - coordinate is measured along the surface and the $\mathrm{y}$ coordinate normal to it. The temperature of the ambient medium is To and the wall temperature is Tw such that Tw $>$ $\mathrm{T} \infty$. The flow along the vertical flat plate contains a species A slightly soluble in the fluid $\mathrm{B}$, the concentration at the plate surface is $\mathrm{CW}$ and the solubility of $\mathrm{A}$ in $\mathrm{B}$ far away from the plate is $\mathrm{C} \infty$. The concentration of dissolved A is small enough. 


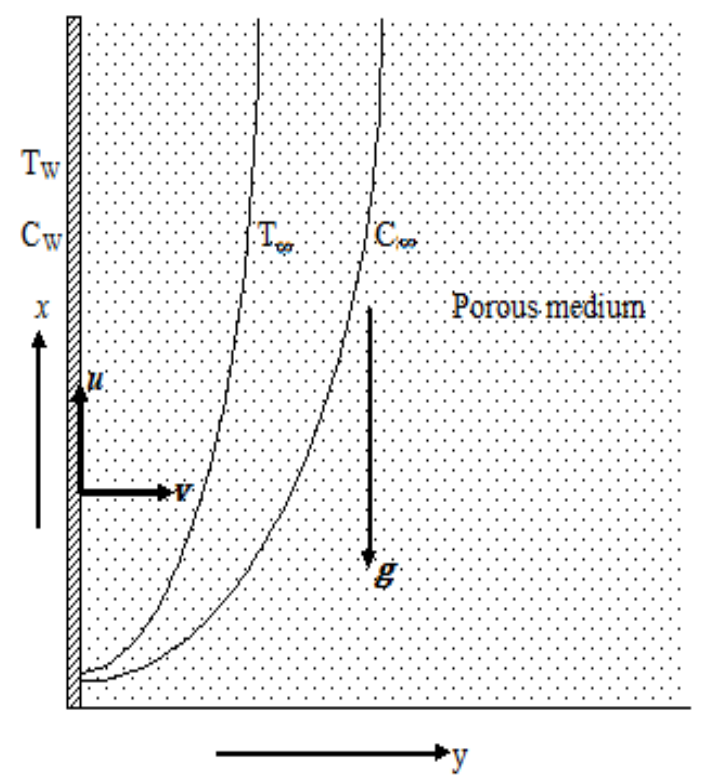

Fig.1: Flow model and physical coordinate system

Under the boundary layer and Boussinesq approximations, the basic boundary layer equations describing the conservation of mass, momentum, energy and concentration can be written as follows:

$$
\frac{\partial u}{\partial x}+\frac{\partial v}{\partial x}=0
$$

$u\left(1+\frac{k \sigma \mu_{e}^{2} H_{0}^{2}}{\mu}\right)=\frac{g k}{v}\left[\beta_{T}\left(T-T_{\infty}\right)-\beta_{C}\left(C-C_{\infty}\right)\right]$

$u \frac{\partial T}{\partial x}+v \frac{\partial T}{\partial y}=\alpha_{m} \frac{\partial^{2} T}{\partial y^{2}}+\frac{D_{m}}{C_{s}} \frac{K_{T}}{C_{P}} \frac{\partial^{2} C}{\partial y^{2}}+\frac{Q_{0}}{\rho C_{P}}\left(T-T_{\infty}\right)$

$u \frac{\partial C}{\partial x}+v \frac{\partial C}{\partial y}=D_{m} \frac{\partial^{2} C}{\partial y^{2}}+\frac{D_{m} K_{T}}{T_{m}} \frac{\partial^{2} T}{\partial y^{2}}-K_{1}\left(C-C_{\infty}\right)^{n}$

where $\mathrm{u}$ and $\mathrm{v}$ are fluid velocity components along the $\mathrm{x}$ and $\mathrm{y}$ axes respectively, $v$ is the kinematic viscosity, $g$ is acceleration due to gravity, $\rho$ is density, $\beta T$ is the coefficient of thermal expansion, $\beta C$ is the coefficient of concentration expansion, $\mathrm{k}$ is the Darcy permeability, KT is the thermal diffusion ratio, $\mathrm{K} 1$ is the dimensional chemical reaction parameter, $\alpha \mathrm{m}$ is thermal diffusivity, Tm is the mean fluid temperature, CS is the concentration susceptibility, C is the concentration, $\mathrm{t}$ is the temperature, $\mathrm{CP}$ is the specific heat at constant pressure, $\mathrm{n}$ is the order of the chemical reaction, Dm is mass diffusivity, $\mu$ is fluid dynamic viscosity, $\sigma$ is electrical conductivity of the fluid.

The boundary conditions are

$$
\left.\begin{array}{lr}
\mathrm{v}=0, \mathrm{~T}=\mathrm{T}_{\mathrm{W}}, \mathrm{C}=\mathrm{C}_{\mathrm{W}} & \text { at } \mathrm{y}=0 \\
\mathrm{~T} \rightarrow \mathrm{T}_{\infty}, \mathrm{C} \rightarrow \mathrm{C}_{\infty} & \text { as } \mathrm{y} \rightarrow \infty
\end{array}\right\}
$$

We now introduce the following dimensionless variables:

$$
\begin{aligned}
& \psi=\alpha_{m} R_{a_{x}}{ }^{\frac{1}{2}} f(\eta), \quad \eta=\left(\frac{y}{x}\right) R_{a_{x}}{ }^{\frac{1}{2}} \\
& \theta=\frac{T-T_{\infty}}{T_{W}-T_{\infty}}, \quad \emptyset=\frac{C-C_{\infty}}{C_{W}-C_{\infty}}
\end{aligned}
$$

where $\psi$ is the stream function that satisfies the continuity equation (1) and is defined as $u=\frac{\partial \psi}{\partial y}, v=\frac{\partial \psi}{\partial x}$ and $R_{a_{x}}=$ $g k \beta_{T}\left(T_{W}-T_{\infty}\right) x /\left(v \alpha_{m}\right)$ is the local Rayleigh number.

Introducing the relation (6) into the equations (2) - (4) we obtain the following governing equations:

$$
\begin{aligned}
&\left(1+M^{2}\right) f^{\prime}=\theta+N \phi \\
& \theta^{\prime \prime}+\frac{1}{2} f \theta^{\prime}+D_{f} \emptyset^{\prime \prime}+\delta \theta=0 \\
& \frac{1}{L e} \emptyset^{\prime \prime}+S r \theta^{\prime \prime}+\frac{1}{2} f \emptyset^{\prime}-\gamma \emptyset^{n}=0
\end{aligned}
$$

where $\mathrm{M}, \mathrm{Le}, \mathrm{D}_{f}, \mathrm{Sr}$ and $\mathrm{N}$ are magnetic, Lewis, Dufour, Soret numbers and the sustentation parameter respectively and are defined as

$$
\left.\begin{array}{c}
M^{2}=\frac{k \sigma \mu_{e}^{2} H_{0}^{2}}{\mu}, \quad L e=\frac{\alpha_{m}}{D_{m}}, \quad D_{f}=\frac{D_{m} K_{T}\left(C_{W}-C_{\infty}\right)}{C_{S} C_{P} \alpha_{m}\left(T_{W}-T_{\infty}\right)}, \\
S r=\frac{D_{m} K_{T}\left(T_{W}-T_{\infty}\right)}{T_{m} \alpha_{m}\left(C_{W}-C_{\infty}\right)}, \quad N=\frac{\beta_{C}\left(C_{W}-C_{\infty}\right)}{\beta_{T}\left(T_{W}-T_{\infty}\right)}
\end{array}\right\}
$$

In order to get the similarity solutions, the constant dimensionless chemical reaction parameter $\gamma$ and heat source parameter $\delta$ was introduced in equation (9) and (8) respectively defined as

$$
\gamma=\frac{K_{1}}{\alpha_{m}} \frac{x^{2}}{R_{a_{x}}}, \quad \delta=\frac{Q_{0}}{\rho C_{p}} \frac{x^{2}}{\alpha_{m} R_{a_{x}}}
$$

The boundary conditions (5) are now transformed to

$$
\left.\begin{array}{ll}
f=0, \theta=1, \varnothing=1 & \text { at } \eta=0 \\
\theta \rightarrow 0, \emptyset \rightarrow 0 & \text { as } \eta \rightarrow \infty
\end{array}\right\}
$$

Equations (7) - (9) are non - linear and so their solution in (4) closed form is not possible. Hence the set of equations (7) (9) under the boundary conditions (12) have been solved numerically with MATLAB's built - in solver bvp4c. From the process of numerical computation, the local Nusselt number and the local Sherwood number, which are respectively proportional to $-\theta^{\prime}(0)$ and $-\emptyset^{\prime}(0)$, are also worked out and their numerical values are presented in a tabular form.

\section{RESULTS AND DISCUSSION}

Numerical calculations have been carried out for various values of the parameters Le, M, N, $\mathrm{D}_{f}, \mathrm{Sr}, \delta, \gamma$ and $n$. Eight cases are considered:

Case I : $\operatorname{Le}=(1,2,4), \mathrm{M}=1, \mathrm{~N}=1, \mathrm{D}_{f}=0.2, \mathrm{Sr}=0.2$, $\gamma=1, n=2, \delta=0.06$

Case II $\quad$ : $\quad \mathrm{Le}=1, \mathrm{M}=(0,0.5,1), \mathrm{N}=1, \mathrm{D}_{f}=0.2, \mathrm{Sr}=$ $0.2, \gamma=1, n=2, \delta=0.06$

Case III : $\mathrm{Le}=1, \mathrm{M}=1, \mathrm{~N}=(1,3,6), \mathrm{D}_{f}=0.2, \mathrm{Sr}=0.2$, $\gamma=1, n=2, \delta=0.06$

Case IV : $\quad$ Le $=1, \mathrm{M}=1, \mathrm{~N}=1, \mathrm{D}_{f}=(0.2,1,3), \mathrm{Sr}=0.2$, $\gamma=1, n=2, \delta=0.06$

Case V : $\quad \mathrm{Le}=1, \mathrm{M}=1, \mathrm{~N}=1, \mathrm{D}_{f}=0.2, \mathrm{Sr}=(0.1,0.2$, $0.5), \gamma=1, n=2, \delta=0.06$

Case VI : $\mathrm{Le}=1, \mathrm{M}=1, \mathrm{~N}=1, \mathrm{D}_{f}=0.2, \mathrm{Sr}=0.2, \gamma=(0$, $0.5,1), n=2, \delta=0.06$ 
Case VII : $\mathrm{Le}=1, \mathrm{M}=1, \mathrm{~N}=1, \mathrm{D}_{f}=0.2, \mathrm{Sr}=0.2, \gamma=1, n=$ $(1,2,3), \delta=0.06$

Case VIII: $\quad$ Le $=1, \mathrm{M}=1, \mathrm{~N}=1, \mathrm{D}_{f}=0.2, \mathrm{Sr}=0.2, \gamma=1, n=$ $2, \delta=(0,0.2,0.3)$

The numerical results for temperature and concentration profiles are displayed in Figures (2)-(17).

Case I:

Figure (2) exhibit temperature profile for various values of Le. It is observed that the temperature decreases exponentially from its maximum value at the plate to its minimum value at the end of the boundary layer. It is also noticed that with an increase in the values of Lewis number Le, the temperature of the binary fluid mixture also increases slightly.

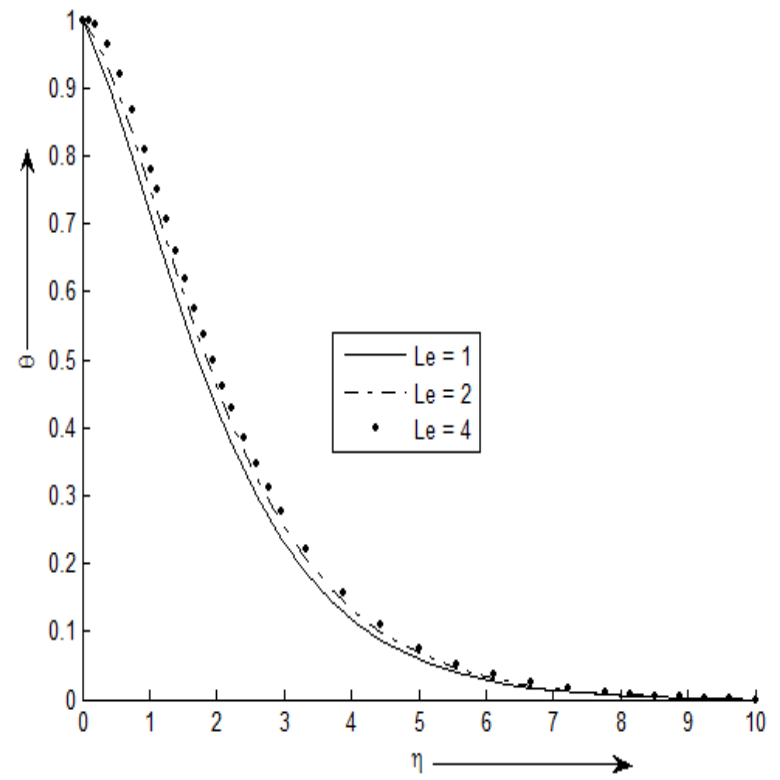

Fig 2: Temperature profile for different values of Le

The concentration profile for different values of Le is shown in figure (3). It is noticed that the concentration decreases exponentially from its maximum value at the plate to its minimum value at the end of the boundary layer. It is also observed that with an increase in the values of Le, the concentration of the fluid decreases sharply.

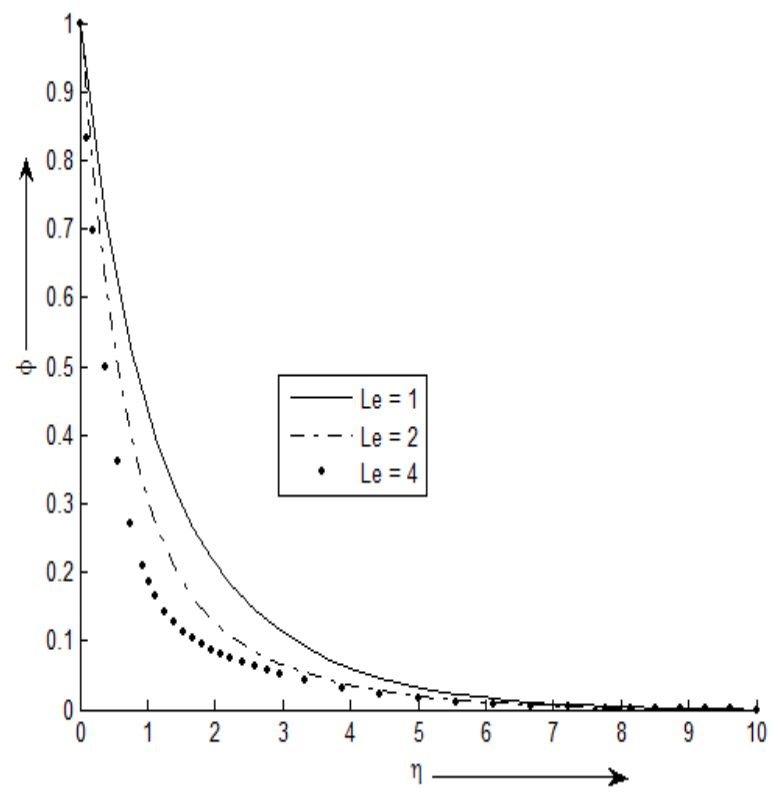

Fig 3: Concentration profile for different values of $\mathrm{Le}$

Case II:

Figure (4) shows the temperature profile for different values of M. It is noticed that there is an exponential decrease in temperature from its maximum value at the plate to its minimum value at the end of the boundary layer. In addition, it depicts that with an increase in the values of the magnetic parameter $\mathrm{M}$, there is also an increase in the temperature of the binary fluid mixture.

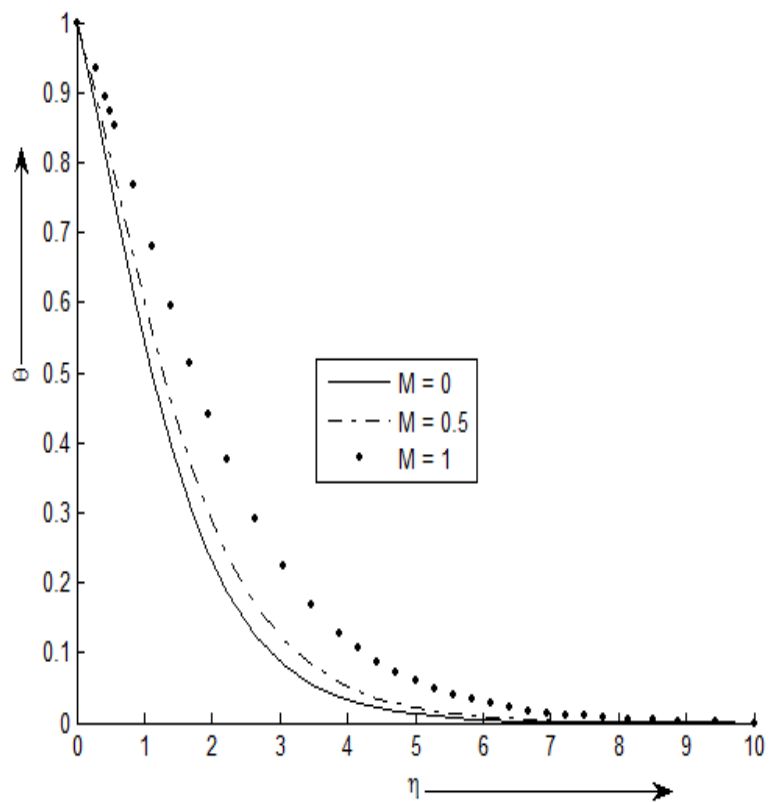

Fig 4: Temperature profile for different values of $M$

The concentration profile for different values of $\mathrm{M}$ is shown in figure (5). It is observed that the concentration decreases exponentially from its maximum value at the plate to its minimum value at the end of the boundary layer. It is also noticed that with an increase in the values of the magnetic parameter $\mathrm{M}$, there is a slight increase in concentration of the rarer and lighter components in the fluid. 


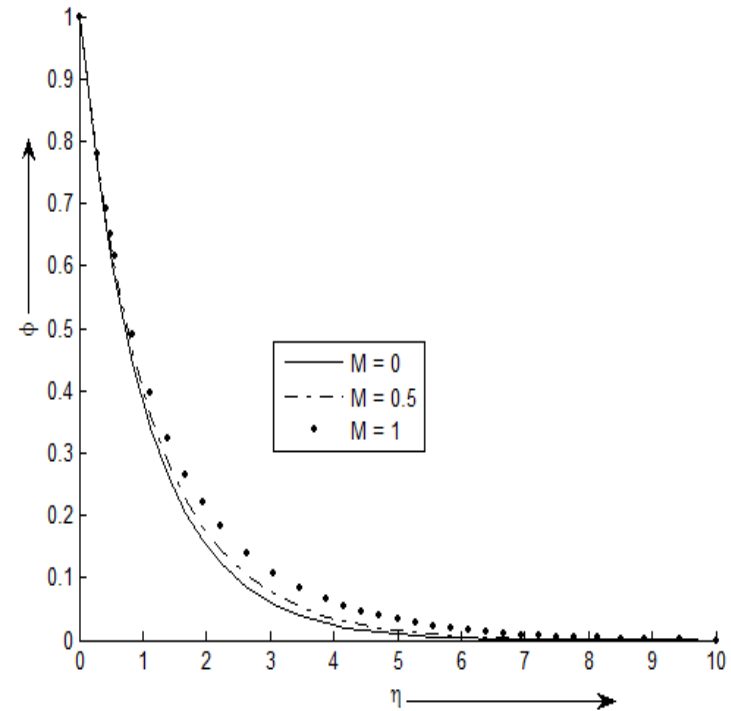

Fig 5: Concentration profile for different values of $M$

\section{Case III:}

Figure (6) depicts the temperature profile for different values of $\mathrm{N}$. It shows that the temperature decreases exponentially from its maximum value at the plate to its minimum value at the end of the boundary layer. It is observed that for various values of sustentation parameter, the temperature varies sharply near the plate. It is also noticed that with an increase in the values of $\mathrm{N}$, the temperature of the fluid decreases sharply.

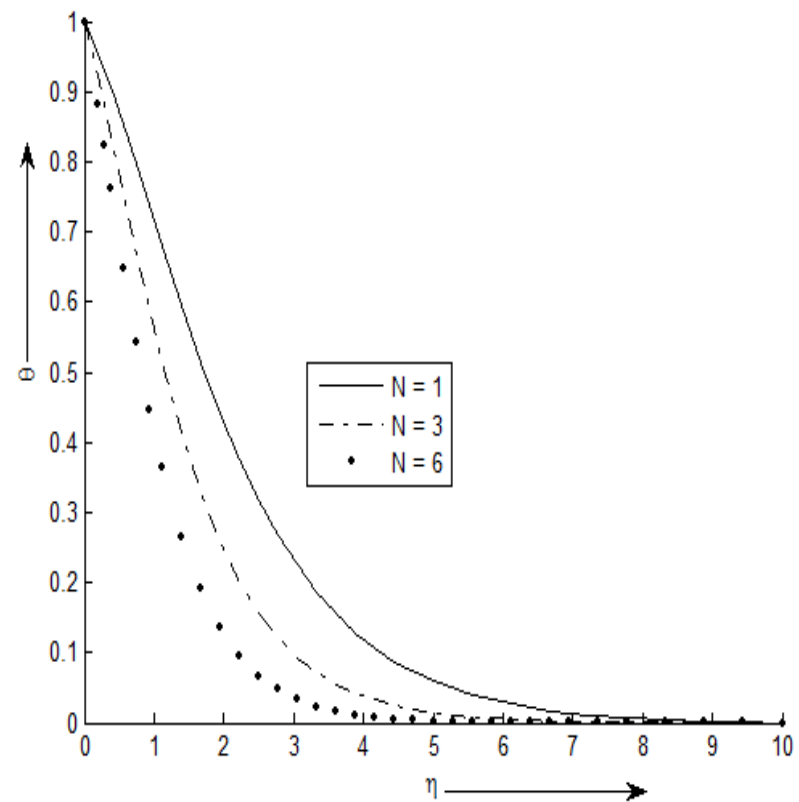

Fig 6: Temperature profile for different values of $\mathbf{N}$

Figure (7) shows the concentration profile for different values of N. It is noticed that there is an exponential decrease in concentration of the rarer and lighter components from its maximum value at the plate to its minimum value at the end of the boundary layer. In addition, it depicts that with an increase in the values of the sustentation parameter $\mathrm{N}$, there is a decrease in the concentration of the binary fluid mixture.

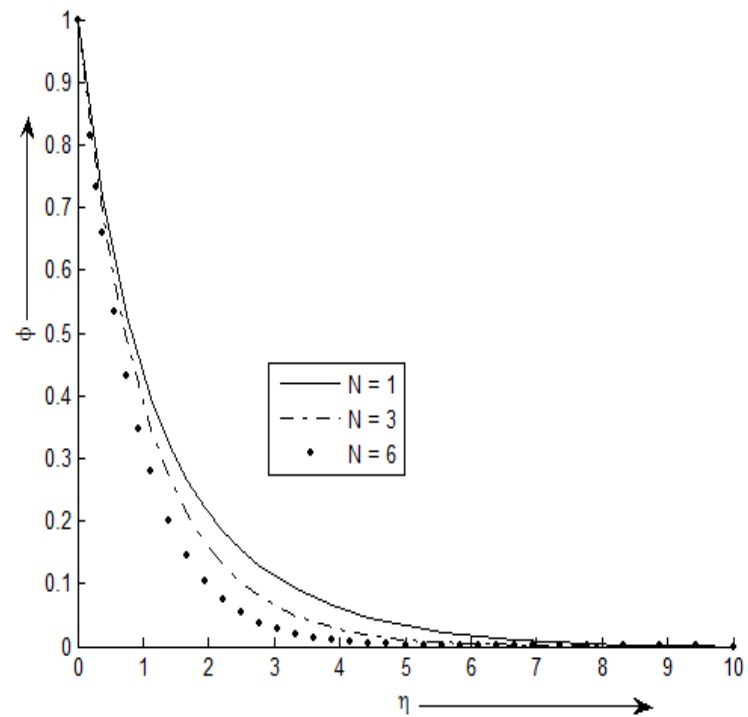

Fig 7: Concentration profile for different values of $\mathbf{N}$ Case IV:

The temperature profile for different values of $\mathrm{D}_{f}$ is shown in figure (8). It is observed that the temperature increases near the plate and attains its maximum value nearly at $\eta=1$ and then decreases exponentially and is minimum at the end of the boundary layer. It is also noticed that for various values of $\mathrm{D}_{f}$, the maximum value of temperature increases rapidly near the wall of the plate as Dufour number increases and attains constant minimum at the end of the boundary layer. It also shows that with an increase in the values of $\mathrm{D}_{f}$, the temperature of the binary fluid mixture increases.

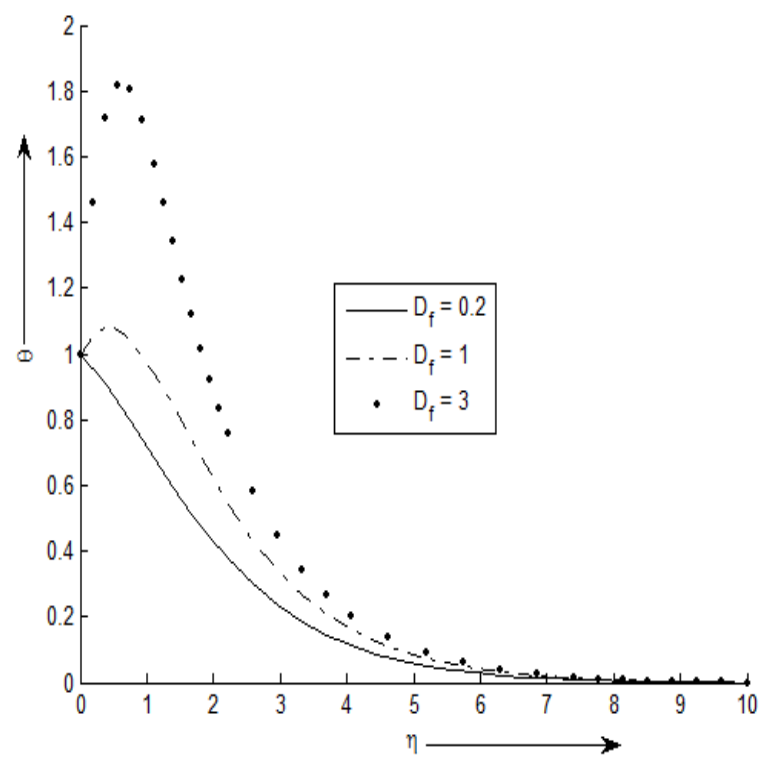

Fig 8: Temperature profile for different values of $D_{f}$

Figure (9) depicts the concentration profile for different values of $\mathrm{D}_{f}$. It shows that the concentration decreases exponentially from its maximum value at the plate to its minimum value at the end of the boundary layer. It is observed that for various values of Dufour number, the concentration varies near the plate. It is also noticed that with an increase in the values of $\mathrm{D}_{f}$ , the concentration of the fluid decreases slightly. 


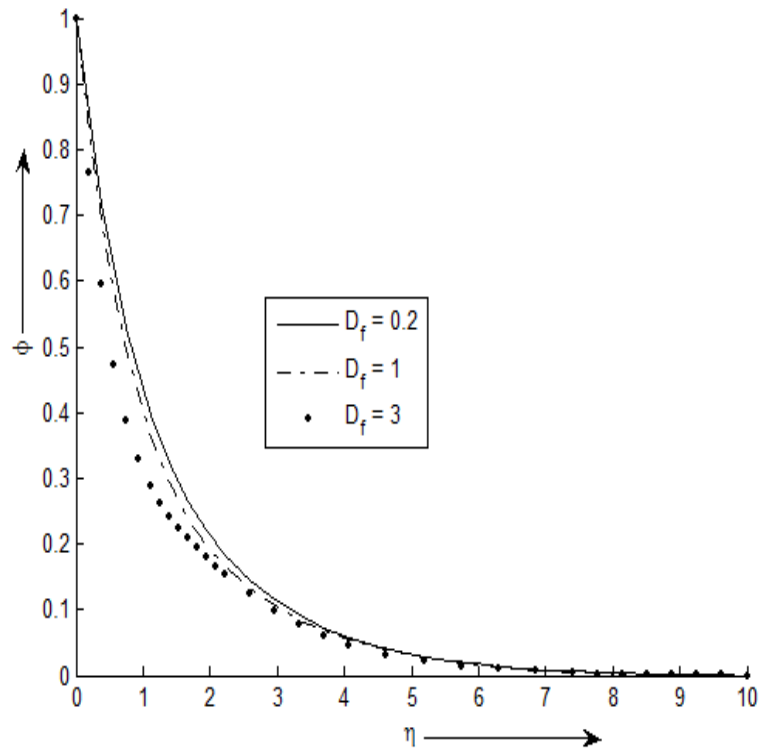

Fig 9: Concentration profile for different values of $D_{f}$

Case V:

Figure (10) exhibit temperature profile for various values of Sr. The temperature decreases exponentially from its maximum value at the plate to its minimum value at the end of the boundary layer. It is observed that there is a slight variation in temperature for different values of Soret number. With the increase in the values of $\mathrm{Sr}$, a decrease in values of temperature is observed.

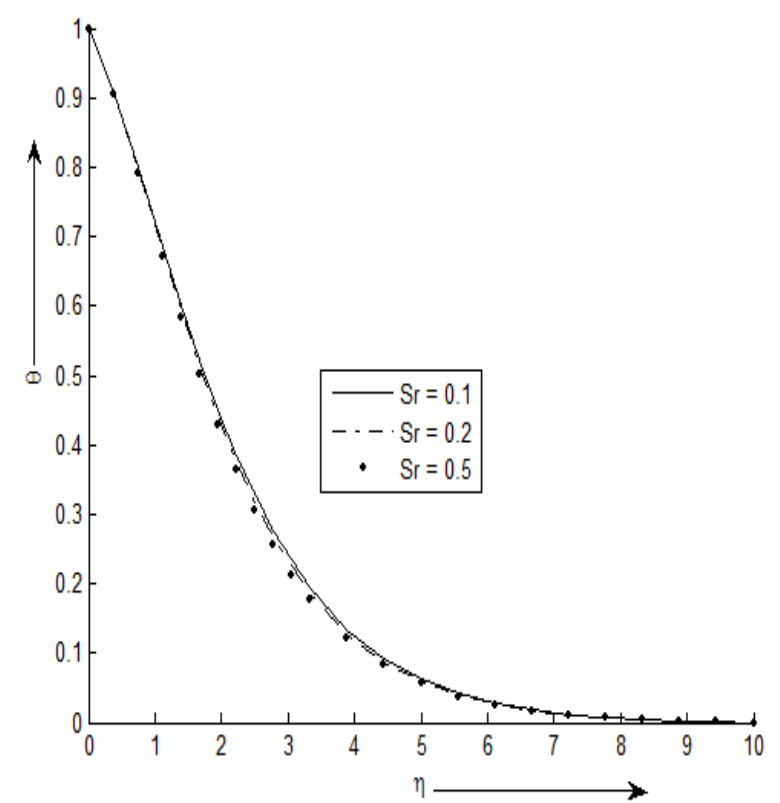

Fig 10: Temperature profile for different values of $\mathrm{Sr}$

The concentration profile for different values of $\mathrm{Sr}$ is shown in figure (11). It is noticed that the concentration decreases exponentially from its maximum value at the plate to its minimum value at the end of the boundary layer. It is also observed that with an increase in the values of $\mathrm{Sr}$, the concentration of the fluid increases.

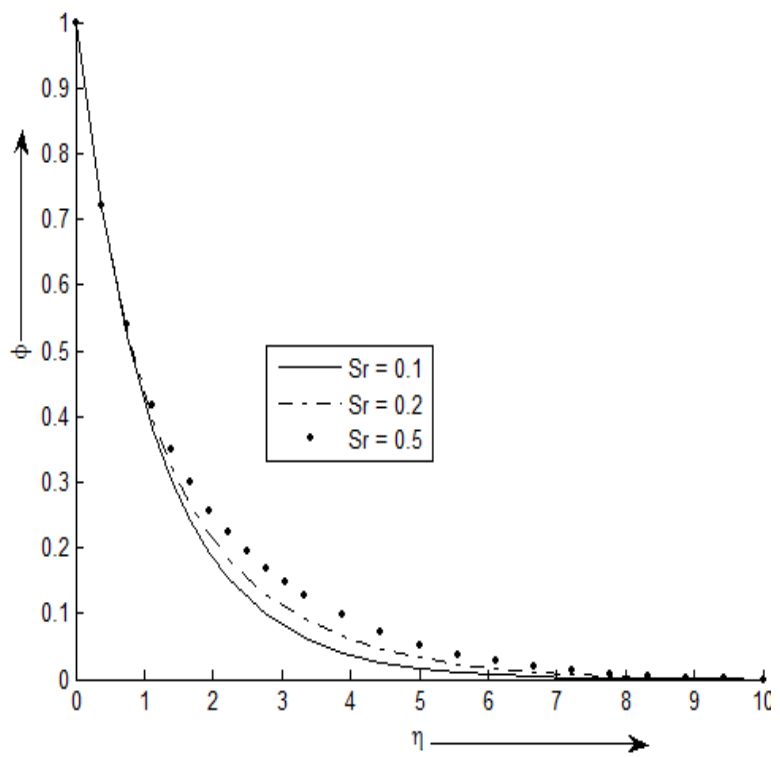

Fig 11: Concentration profile for different values of $\mathrm{Sr}$

Case VI:

The temperature profile for different values of $\gamma$ is shown in figure (12). It shows that the temperature decreases exponentially from its maximum value at the plate to its minimum value at the end of the boundary layer. It is observed that there is a very slight variation in temperature for different values of $\gamma$. It is also noticed that, with the increase in the values of the chemical reaction parameter, an increase in values of temperature is observed.

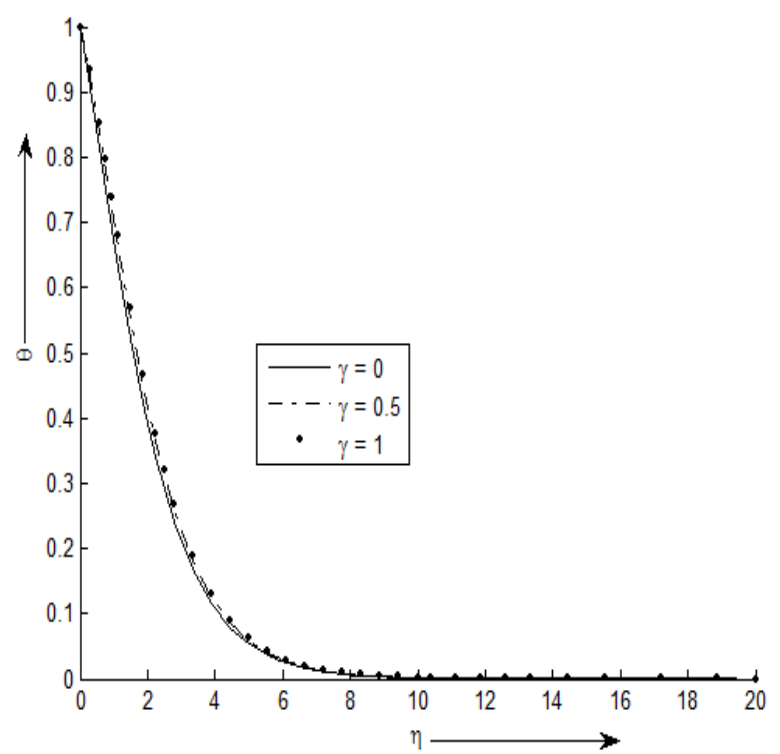

Fig 12: Temperature profile for different values of $\gamma$

Figure (13) depicts the concentration profile for different values of $\gamma$. It shows that the concentration decreases exponentially from its maximum value at the plate to its minimum value at the end of the boundary layer. It is observed that for various values of $\gamma$, the concentration varies near the plate. It is also noticed that with an increase in the values of $\gamma$, the concentration of the fluid decreases. 


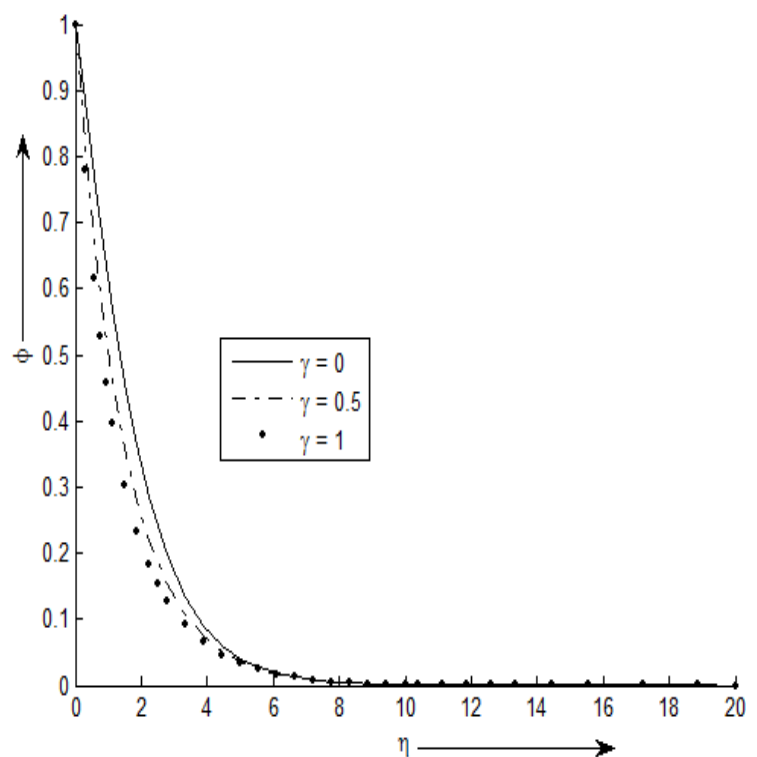

Fig 13: Concentration profile for different values of $\gamma$

\section{Case VII:}

Figure (14) depicts the temperature profile for different values of $n$. It is noticed that there is an exponential decrease in temperature from its maximum value at the plate to its minimum value at the end of the boundary layer. In addition, it shows that with an increase in the values of the order of the chemical reaction $n$, there is a decrease in the temperature of the binary fluid mixture.

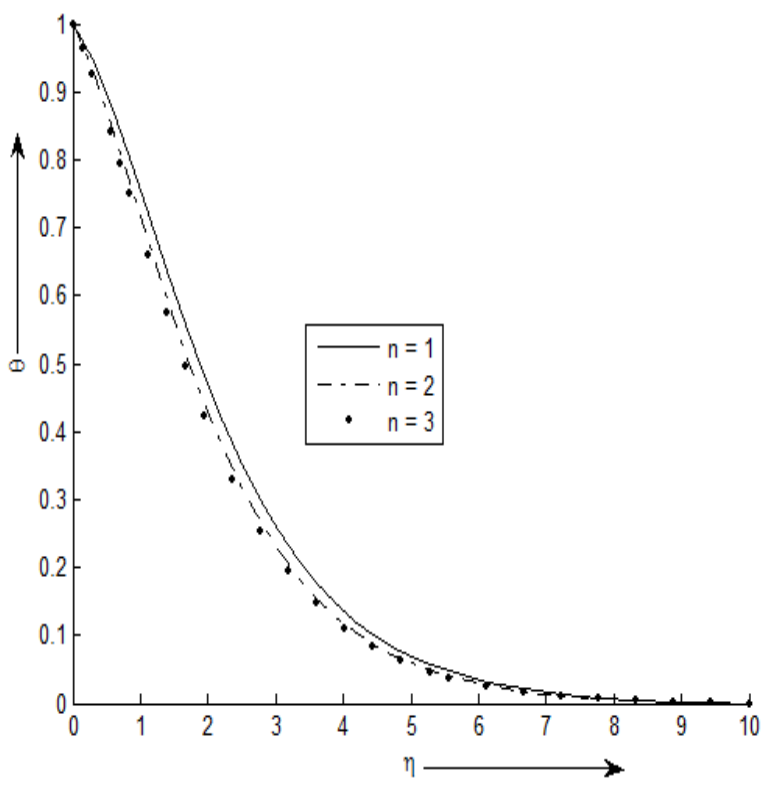

Fig 14: Temperature profile for different values of $\mathbf{n}$

The concentration profile for different values of $n$ is shown in figure (15). It is noticed that the concentration decreases exponentially from its maximum value at the plate to its minimum value at the end of the boundary layer. It is also observed that with an increase in the values of $n$, the concentration of the binary fluid mixture increases sharply.

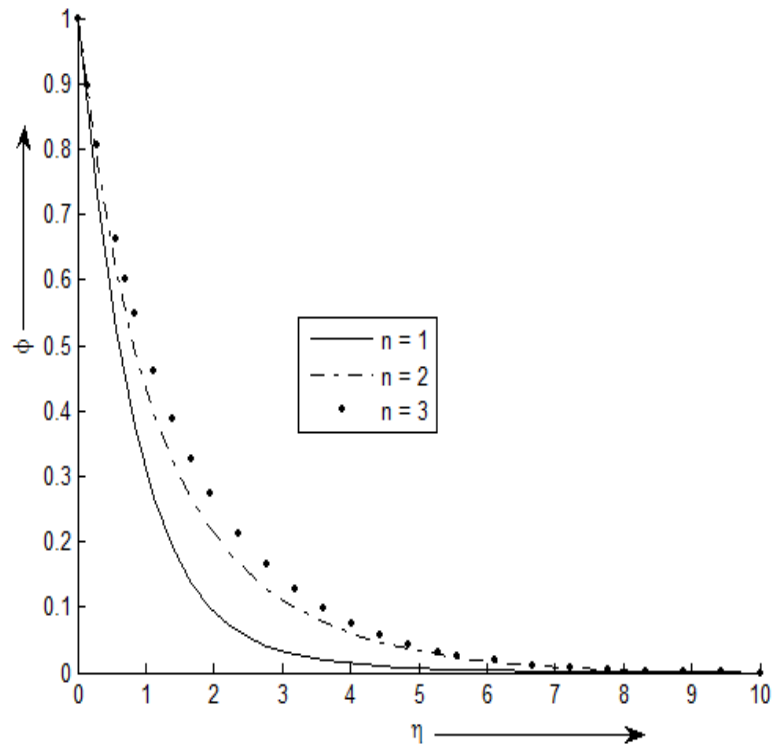

Fig 15: Concentration profile for different values of $\mathbf{n}$

Case VIII:

Figure (16) exhibit temperature profile for various values of $\delta$. It is observed that the temperature increases near the plate and attains its maximum value nearly at $\eta=1$ and then decreases exponentially and is minimum at the end of the boundary layer. It is also noticed that for various values of $\delta$, the maximum value of temperature increases rapidly near the wall of the plate as heat source parameter increases and attains constant minimum at the end of the boundary layer. It also shows that with an increase in the values of $\delta$, the temperature of the binary fluid mixture increases.

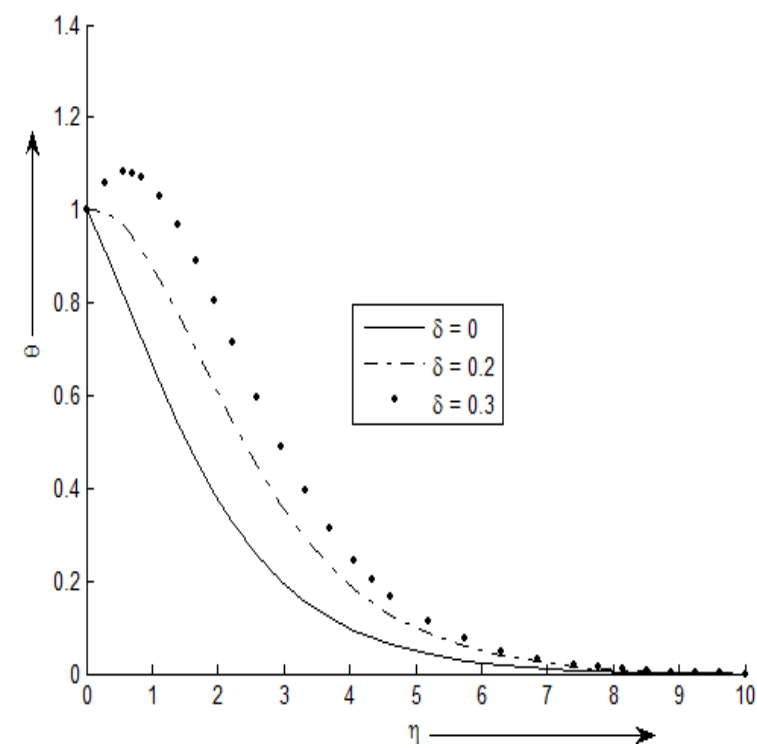

Fig 16: Temperature profile for different values of $\delta$

The concentration profile for different values of $\delta$ is shown in figure (17). It is noticed that the concentration decreases exponentially from its maximum value at the plate to its minimum value at the end of the boundary layer. It is also observed that with an increase in the values of heat source parameter $\delta$, the concentration of rarer and lighter components of the binary fluid mixture decreases.

Finally, the effects of the rate of heat and mass transfer are shown in Table -1 . The behaviour of these parameters is self 
- evident from the Table - 1 and hence further discussion

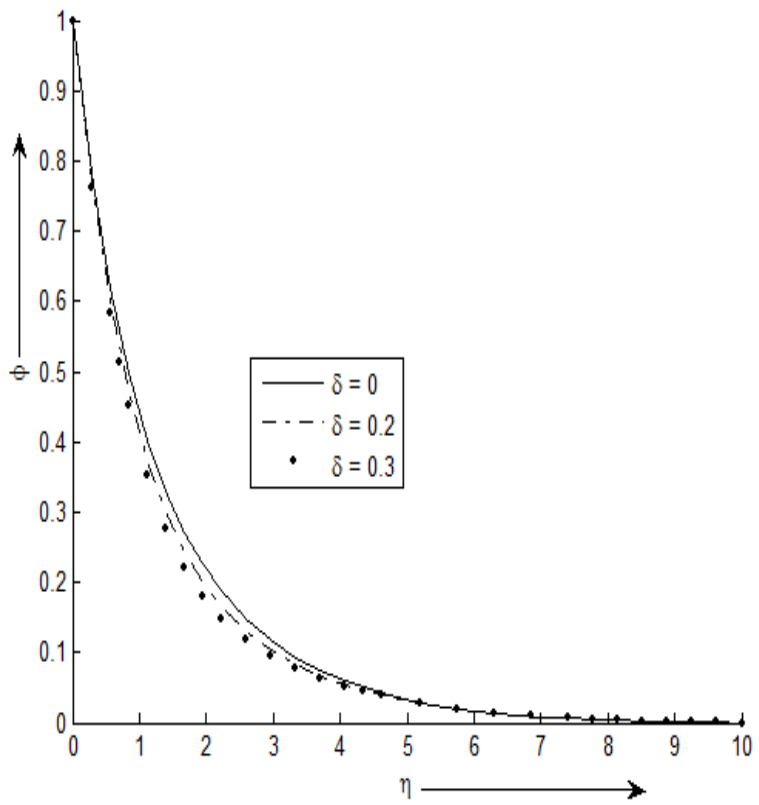

Fig 17: Concentration profile for different values of $\delta$

Table - 1. Numerical values for $\theta^{\prime}(0)$ and $\Phi^{\prime}(0)$.

\begin{tabular}{|c|c|c|c|c|c|c|c|c|c|}
\hline $\mathbf{M}$ & $\mathbf{D}_{f}$ & $\mathbf{L e}$ & $\boldsymbol{\gamma}$ & $\boldsymbol{\delta}$ & $\mathbf{n}$ & $\mathbf{S r}$ & $\mathbf{N}$ & $-\boldsymbol{\theta}^{\prime}(\mathbf{0})$ & $-\boldsymbol{\Phi}^{\prime}(\mathbf{0})$ \\
\hline 0 & 0.2 & 1 & 1 & 0.06 & 2 & 0.2 & 1 & 0.4224 & 1.0010 \\
\hline 0.5 & 0.2 & 1 & 1 & 0.06 & 2 & 0.2 & 1 & 0.3480 & 0.9707 \\
\hline 1 & 0.2 & 1 & 1 & 0.06 & 2 & 0.2 & 1 & 0.2085 & 0.9252 \\
\hline 1 & 0.2 & 1 & 1 & 0.06 & 2 & 0.2 & 1 & 0.2085 & 0.9252 \\
\hline 1 & 1 & 1 & 1 & 0.06 & 2 & 0.2 & 1 & -0.4271 & 1.0253 \\
\hline 1 & 3 & 1 & 1 & 0.06 & 2 & 0.2 & 1 & -1.0253 & 1.4816 \\
\hline 1 & 0.2 & 1 & 1 & 0.06 & 2 & 0.2 & 1 & 0.2085 & 0.9252 \\
\hline 1 & 0.2 & 2 & 1 & 0.06 & 2 & 0.2 & 1 & 0.1079 & 1.3477 \\
\hline 1 & 0.2 & 4 & 1 & 0.06 & 2 & 0.2 & 1 & -0.0384 & 2.0093 \\
\hline 1 & 0.2 & 1 & 0 & 0.06 & 2 & 0.2 & 1 & 0.3267 & 0.4244 \\
\hline 1 & 0.2 & 1 & 0.5 & 0.06 & 2 & 0.2 & 1 & 0.2568 & 0.7151 \\
\hline 1 & 0.2 & 1 & 1 & 0.06 & 2 & 0.2 & 1 & 0.2074 & 0.9252 \\
\hline 1 & 0.2 & 1 & 1 & 0 & 2 & 0.2 & 1 & 0.2902 & 0.9135 \\
\hline 1 & 0.2 & 1 & 1 & 0.2 & 2 & 0.2 & 1 & -0.0410 & 0.9600 \\
\hline 1 & 0.2 & 1 & 1 & 0.3 & 2 & 0.2 & 1 & -0.2869 & 0.9937 \\
\hline 1 & 0.2 & 1 & 1 & 0.06 & 1 & 0.2 & 1 & 0.1527 & 1.1142 \\
\hline 1 & 0.2 & 1 & 1 & 0.06 & 2 & 0.2 & 1 & 0.2085 & 0.9252 \\
\hline 1 & 0.2 & 1 & 1 & 0.06 & 3 & 0.2 & 1 & 0.2367 & 0.8218 \\
\hline 1 & 0.2 & 1 & 1 & 0.06 & 2 & 0 & 1 & 0.2059 & 0.9215 \\
\hline 1 & 0.2 & 1 & 1 & 0.06 & 2 & 0.2 & 1 & 0.2085 & 0.9252 \\
\hline 1 & 0.2 & 1 & 1 & 0.06 & 2 & 0.5 & 1 & 0.2111 & 0.9337 \\
\hline 1 & 0.2 & 1 & 1 & 0.06 & 2 & 0.2 & 1 & 0.2085 & 0.9252 \\
\hline 1 & 0.2 & 1 & 1 & 0.06 & 2 & 0.2 & 3 & 0.4026 & 0.9949 \\
\hline 1 & 0.2 & 1 & 1 & 0.06 & 2 & 0.2 & 6 & 0.6170 & 1.1008 \\
\hline
\end{tabular}

\section{CONCLUSION}

Concentration of the rarer and lighter component of the binary fluid mixture increases with the increase of Magnetic parameter, Soret number and the order of chemical reaction parameter whereas decreases with the increase of the Lewis number, sustentation parameter, Dufour number and heat source parameter.

In this paper we have considered the permeability of the porous medium to be constant. In many cases permeability of the porous media is found to be variable. So further investigation of the problem can be done to study the effects of the variation of the permeability of the porous medium as the concentration of the components of the binary fluid mixture.

\section{REFERENCES}

[1] Nield, D.A. and Bejan, A. 2006. Convection of Porous Media, $3^{\text {rd }}$ edition, Springer, New York.

[2] Ingham, D.B. and Pop, I. 1998. Transport Phenomena in Porous Media I, Pergamon, Oxford.

[3] Ingham, D.B. and Pop, I. 2002. Transport Phenomena in Porous Media II, Pergamon, Oxford. 
[4] Bejan, A. and Khair, K.R. 1985. "Heat and Mass transfer by natural convection in a porous medium", Int. J. Heat Mass Transfer, 28 , pp. 909-918.

[5] Makinde, O.D. 2005. "Free-convection flow with thermal radiation and mass transfer past a moving vertical porous plate", Int. Comm. Heat Mass transfer, 32, pp. 14111419.

[6] Makinde, O.D. and Ogulu, A. 2008. " The effect of Thermal Radiation on Heat and Mass transfer Flow of a variable viscosity Fluid past a Vertical Porous plate permeated by a Transverse Magnetic Field", Chem. Eng. Comm., 195(12), pp. 1575-1584.

[7] Ibrahim, S.Y. and Makinde, O.D. 2011. "Radiation effect on chemically reacting magnetohydrodynamics (MHD) boundary layer flow of heat and mass transfer through a porous vertical flat plate", Int. J. Phys. Sci, 6(6), pp. 1508-1516.

[8] Soret, C. 1880. "Influence de la temperature sur la distribution des sels dans leurs solutions". C. R. Acad Sci Paris, 91, pp. 289-291.

[9] Kafoussias, N. G. and Williams, E. M. 1995. "Thermaldiffusion and diffusion-thermo effects on mixed freeforced convective and mass transfer boundary layer flow with temperature dependent viscosity", Int. J. Eng. Sci., 33, pp. 1369-1384.

[10] Anghel, M., Takhar, H.S. and Pop, I. 2000. "Dufour and Soret effects on free convection boundary layer over a vertical surface imbedded in a porous medium", Studia Universitatis Babes-Bolyai. Mathematica, XIV(4), pp. 11-21.

[11] Postelnicu, A. 2004. "Influence of a magnetic field on heat and mass transfer by natural convection from vertical surface in porous media considering Soret and Dufour effects", Int. J. Heat and Mass Transfer, 47, pp. 1467-1472.
[12] Alam, M.S. and Rahman, M.M. 2006. "Dufour and Soret effects on Mixed Convection Flow Past a Vertical Porous Flat Plate with Variable Suction" Nonlinear Analysis: Modelling and Control, 11(1), pp. 3-12.

[13] Sharma, B.R. 2011. "Soret and Dufour effects on Separation of a Binary fluid mixture in MHD natural convection flow in porous media", Mathematical Forum, XXIV, pp. 17-27.

[14] Chambre, P.L. and Young, J.D. 1958. "On the diffusion of a Chemically reactive species in a laminar boundary layer flow". The Physics of Fluids, vol. 1, pp. 48-54.

[15] Apelblat, A. 1980. Mass Transfer with a chemical reaction of the first order, The Chemical Engineering Journal, vol. 19, pp. 19-37.

[16] Das, U.N., Deka, R.K. and Soundalgekar, V.M. 1994 "Effects of Mass Transfer on flow past an Impulsively started infinite vertical plate with constant Heat flux and chemical Reaction”, Forschung in Ingenieurwsen, 60, pp. 284-287.

[17] Das, U.N., Deka, R.K. and Soundalgekar, V.M. 1999 "Effects of Mass Transfer on flow past a moving isothermal vertical plate in the presence of chemical reaction", The Bulletin, GUMA, 5, pp. 13-20.

[18] Postelnicu, A. 2007. "Influence of Chemical reaction on heat and mass transfer by natural covection from vertical surfaces in porous media considering Soret and Dufour effects", Heat and Mass transfer, 43, pp. 595-602.

[19] Jyothi Bala, A. and Vijaya Kumar Varma, S. 2011. "Unsteady MHD Heat and Mass transfer flow past a semi vertical porous moving plate with variable suction in the presence of heat generation and homogeneous chemical reaction." Int. J. of Appl. Math and Mech. 7(7), pp. 20 44 